Canadian Oncology

Nursing Journal

Revue canadienne

de soins infirmiers

en oncologie

Volume 28, Issue 3 • Summer 2018

elSSN: 2368-8076 


\title{
Patient-reported care domains that enhance the experience of "being known" in an ambulatory cancer care centre
}

\author{
by Chloe Grover, Erin Mackasey, Erin Cook, Lucie Tremblay, and Carmen G. Loiselle
}

\section{ABSTRACT}

Purpose: This study explored patients' perceptions of "being known" in an ambulatory chemotherapy unit.

Methods: Using a qualitative descriptive design, 10 participants with various cancer diagnoses were recruited from a large cancer centre in Montreal, Quebec. Audiotaped individual interviews were transcribed verbatim. Textual data were coded and analyzed thematically.

Findings: Participants spoke of their need to have the staff approach them as individuals first and then as persons with cancer. They further underscored the importance of: (1) feeling truly welcome in the cancer care environment, (2) being provided with person-and situation-responsive care, and (3) considering occupational and social roles that go beyond the "sick role". Mutual patient-nurse disclosure also contributed to perceptions of a personalized care approach.

Implications for nursing: In addition to key elements construed as crucial for enhancing perceptions of being known, future studies should further document how the interplay among demographic, physical/psychological, and cultural factors affect these perceptions.

\section{ABOUT THE AUTHORS}

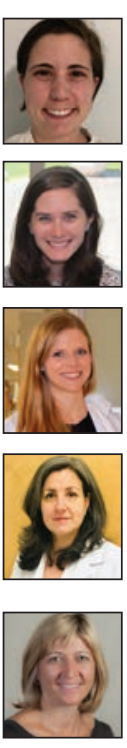

Chloe Grover, RN, MSc, Ingram School of Nursing, McGill

University, Montreal, QC

Erin Mackasey, RN, MSc, Ingram School of Nursing, McGill

University, Montreal, QC

Erin Cook, RN, MSc, Head Nurse, Oncology Clinic, CIUSSS

du Centre-Ouest-de-l'île-de-Montréal, Oncology Clinic, Segal

Cancer Centre, Montreal, QC

Lucie Tremblay, RN, BScN, Nurse Clinician, Assistant Head

Nurse, CIUSSS du Centre-Ouest-de-l'île-de-Montréal, Oncology

Clinic, Segal Cancer Centre, Jewish General Hospital, Montréal,

QC

Carmen G. Loiselle*, RN, PhD, Professor, Department of

Oncology and Ingram School of Nursing, McGill University,

Co-Director (Academic) and Senior Investigator, Segal Cancer

Centre, Jewish General Hospital, 3755 Côte-Sainte-Catherine

Rd, Pav. E-748, Montreal, QC H3T 1E2

514-340-8222 ext. 23940; carmen.g.loiselle@mcgill.ca

*corresponding author

DOI:10.5737/23688076283166171

\section{BACKGROUND}

A cancer diagnosis often has a profound impact on patients' $A$ and families' lives. For individuals receiving cancer treatment, the primary nurse is often the main source of professional guidance and support. Providing this type of care requires an in-depth understanding of the physical and psychosocial needs and preferences of patients.

\section{Person-Centred Care (PCC)}

Person-centred care has been defined as care that is respectful and responsive to individual patient preferences, needs, and values, and ensures that a patient's values guide all clinical decisions (Balogh et al., 2011). Similarly, patients as partners (PP) in care is a recent concept that regards patients as integral units of their healthcare team (Pomney, Ghadiri, Karazivan, Fernandez \& Clavel, 2015). To meet patients' needs, it is widely recognized that PCC and PP movements are paramount for cancer care to be truly comprehensive (Arora, Street, Epstein, \& Butow, 2009; Balogh et al., 2011; National Cancer Institute, 2007; Kvåle $\&$ Bondevik, 2008). When operationalized, this means listening to patients' questions, needs, and views; sharing these with other members of the healthcare team; providing affected individuals with timely cancer-related information and supporting goals that are in accordance with patients' wishes and abilities (Flagg, 2015). When implemented, these approaches lead to higher patient satisfaction and trust in their healthcare team (Thórarinsdóttir \& Kristjánsson, 2013).

\section{"Being Known"}

"Being known" is defined by Thorne et al. (2005) as a component of quality care that addresses the unique characteristics and needs of patients with a focus on human connections. This concept is increasingly becoming central to the delivery of person-centred care through recognition, understanding and acting in line with patients' personal preferences and through acknowledging one's personhood. (Fillion, de Serres, Cook, Goupil, Bairati, \& Doll, 2009; Jacobsen, Bouchard, Emed, Lepage, \& Cook 2015; Thorne, Kuo, Armstrong, McPherson, Harris, \& Hislop, 2005).

Individualized care in the cancer setting occurs when healthcare providers view the patient as unique and orient their care as a function of patients' goals, feelings, and experiences, allowing them to feel connected with the healthcare team (Fillion et al., 2009; S. Jacobsen et al., 2015). Unfortunately, research has shown that patients within cancer care settings often feel as though their needs are not identified and their personal concerns are rarely addressed (Brataas, Thorsnes, \& Hargie, 2010). In response, clinicians can enhance the patient experience by being attentive to goals and needs, acting in line 


\begin{tabular}{|c|c|}
\hline & $n$ \\
\hline \multicolumn{2}{|l|}{ Gender } \\
\hline Male & 6 \\
\hline Female & 4 \\
\hline \multicolumn{2}{|l|}{ Age } \\
\hline $20-40$ years & 1 \\
\hline $41-60$ years & 2 \\
\hline $61-80$ years & 6 \\
\hline $81-90$ years & 1 \\
\hline \multicolumn{2}{|l|}{ Type of Cancer (Primary) } \\
\hline Testicular & 1 \\
\hline Colorectal & 4 \\
\hline Breast & 2 \\
\hline Renal & 1 \\
\hline Hematological & 2 \\
\hline \multicolumn{2}{|l|}{ Stage of Cancer } \\
\hline Stage I & 0 \\
\hline Stage II & 2 \\
\hline Stage III & 3 \\
\hline Stage IV & 3 \\
\hline Unknown & 2 \\
\hline \multicolumn{2}{|l|}{ Time Since Diagnosis } \\
\hline$<6$ months & 3 \\
\hline $6-12$ months & 3 \\
\hline$>12-24$ months & 3 \\
\hline$>24$ months & 1 \\
\hline \multicolumn{2}{|c|}{ Approximate Number of Clinic Visits to Date } \\
\hline$<5$ Visits & 1 \\
\hline 5-10 Visits & 5 \\
\hline $11-15$ Visits & 0 \\
\hline $16-20$ Visits & 4 \\
\hline \multicolumn{2}{|l|}{ Treatment } \\
\hline Currently Receiving Treatment & 9 \\
\hline Treatment Completed & 1 \\
\hline
\end{tabular}

with patients' beliefs and values, and being attuned to other aspects of a patient's life(Kruijver, Kerkstra, Bensing, \& van de Wiel, 2000; Mazor et al., 2013; Thomsen, Pedersen, Johansen, Jensen, \& Zachariae, 2007). As a key member of the healthcare team, nurses play an important role in providing individualized care to patients throughout the cancer journey. This study explores how patients experience "being known" in a busy ambulatory chemotherapy setting.

\section{METHOD}

\section{Study Design, Sample, and Setting}

To address the main goals of the study, rich descriptions of the phenomenon of interest were sought. For this, a qualitative descriptive design was used, allowing for an in-depth exploration of the topic. The interview guide developed by the authors contained open-ended questions to elicit patients' experiences. The interview questions were developed using previous "being known" literature, as well as research on nurse-patient interactions. Examples of questions included: "What does your nurse do that makes you feel that she/he really knows you?", "What has your experience been with having your nurse acknowledge you as a unique person?", and "Do you feel that your care has been adapted according to your needs?"

Participants were recruited from an ambulatory University-affiliated cancer centre in Montréal, Québec, from September 1, 2016 to December 31, 2016. Convenience sampling was used to recruit ten participants and inclusion criteria were as follows: Patients who were (1) currently or had previously been receiving cancer treatment at the centre, (2) age 18 years or older, and (3) able to converse and read English or French. Patients were excluded if they: (1) were followed by staff from the Clinical Research Unit, or (2) had a cognitive or physical limitation (e.g., extreme pain or nausea), that prevented them from fully participating in the study. Of the 20 participants initially recruited, three were unreachable (after three attempts to contact them), three cancelled the appointment, one was excluded due to speaking neither French nor English, and three participants' interviews were not completed before the academic deadline. The resulting sample $(\mathrm{N}=10)$ consisted of patients with cancer who were currently $(n=9)$ or had previously $(\mathrm{n}=1)$ received treatment in the ambulatory cancer care centre (please see Table 1 for demographic data). The sample consisted of males $(n=6)$ and females $(n=4)$ who had various cancer diagnoses, including: breast $(n=2)$, colorectal $(n=4)$, hematological $(n=2)$, testicular $(n=1)$, and renal $(n=1)$. Participants ranged in age from 20 to 90 years, with three participants being $\leq 60$ years old and seven participants older than 60. All participants reported that they had never previously been treated for cancer. The time since current diagnosis was reported as less than six months $(n=3)$, six to 12 months $(n=3), 13$ to 24 months $(n=3)$, and 15 years $(n=1)$. Of note, this ambulatory setting utilizes a primary care approach, whereby a nurse is assigned to a patient during active cancer treatment. In the absence of the primary nurse on certain days of the week, a different nurse is responsible for the delivery of care.

Participants were recruited by community cancer support volunteers or researchers, who briefly introduced the study 
to eligible patients and obtained their permission for the researchers to contact them. Researchers met with interested patients, reviewed the consent form, and after consent, conducted an in-depth interview at a mutually agreed upon time and location.

\section{Data Collection and Analysis}

Information was collected through the use of semi-structured, face-to-face interviews that were audio recorded and participants filled out a sociodemographic questionnaire following the interview. Transcription of the audio recorded interviews occurred within one week of the interview. Member checking was done by the authors during participant interviews to clarify the data obtained and to confirm accuracy in the interpretation of participants' responses. Following the interview, participants were asked to restate or clarify their thoughts on the answers they provided. This debriefing verified interviewers' understanding of the issues reported and ensured credibility and dependability of the data collected.

\section{STUDY FINDINGS}

Thematic analysis was used to categorize key elements pertaining to how participants described "being known." Grouping data into themes facilitated analysis and allowed for more accurate representation of findings (Boyatzis, 1998).

Intitially, participants struggled to articulate what contributed to their perceptions of "being known". For instance, they often asked to repeat the question or elaborate on what interviewers meant. Many then used various concrete actions to illustrate their experiences. From these experiences, significant statements of meaningful interactions emerged. Verbatim transcripts were subsequently reviewed according to these examples, and commonalities and differences were highlighted. Similar meanings were abstracted, which led to the formulation of three larger themes: (1) Feeling truly welcome in the cancer care environment, (2) Being provided with person- and situation-responsive care, and (3) Being acknowledged as a person with occupational and social interests that go beyond the "sick role."

\section{Feeling Truly Welcome in the Cancer Care Environment}

Feeling welcome was described in various ways, including the general attitude of healthcare team members, positive interactions with nurses and volunteers, and not "feeling like a number." Positive attitudes of the healthcare team included an open, friendly, caring, and respectful approach, which were valued by the participants and contributed to their perceptions of "being known". A personable attitude was described as familiarity and greetings. As stated by one participant: "[nurses] recognize my face when I come in, I recognize their face" (P7). Similarly, another underscored that feeling "known" can be as basic as being friendly: "Smile for people, talk a little bit, know who they are and a good hello and everything goes well" (P15, translated from French).

Another described how the attitudes of the nursing staff contributed to feeling cared for: “... it's personal, a warmth and stuff, so I feel like I'm in caring hands with my nurse” (P5). Several patients perceived feeling welcome in the cancer care environment by being able to share personal stories with their primary nurse and having the nurse share, as well. When asked about what was important in "being known" by her nurse, one participant stated: "Well, you know, asking about the family, you know, sharing about her family, stories, ah, just being a bit more personal, I guess, knowing a bit more about me and me knowing about her" (P5). Another participant expressed how her perception of "being known" was enhanced through knowing about the nurses' background and personal histories (P2). Sharing similar family experiences was also important for some participants in feeling personally connected to their nurse.

Participant 1 articulated this experience as: "she's a person, like the patient is a person." More specifically, a participant provided an example of developing a connection with her nurse's family and talking about their children during each clinic visit: "[my nurse] has little boys and she'll mention them and she knows that I have one, so it's, you know, ongoing" (P5). Similarly, another participant felt better able to connect with his healthcare team when they spoke the same language as him. He noted this by saying: "with [my nurse] I can explain more things. There are also two other Latina nurses here that I speak with and they reassure me and are always open with me and that's nice" (P19, translated from French).

Healthcare providers seeing the patient as unique, as opposed to being made to feel like a number, was discussed in relation to the extent to which participants felt welcome in the cancer care environment. In defining how he perceived "being known," a participant contrasted his previous experience elsewhere of "feeling like a number" with his current interactions: "I walked in there and I felt like I was at a cattle call, it was so impersonal, very modern and fancy and everything, but sure, I felt like a number over there... that it's not a number here, that it's more personal, and that it's not just trying to move people through as fast as possible... In spite of them still trying to move just as fast as everybody everywhere else, there just seems to be a more personable thing here. Like they definitely, you know, it's just the feeling when you walk in the place, it doesn't feel the same" (P11).

This sentiment and its impact was also highlighted by another participant “... she's not treating me as a number, she knows my personal history and, well, that's very reassuring” (P7).

\section{Being Provided with Person- and Situation-Responsive Care}

In this study, person-responsive care refers to how healthcare providers adjust to changes in patients' physical and psychosocial needs. The majority of participants $(n=9)$ reported person- and situation-responsive care as key to "being known." This was described through examples of how the healthcare team tailored the treatment plan to the patient's situation, communication being seamless for all involved, and how comprehensive, tailored care was provided.

Participants described how coordination of the treatment plan reflected their needs and treatment situation. A participant described how the nurse adapted his care with respect to managing symptoms and treatment at home: “... when you have your bottle that you go home with she hooked it up so it would be in the inside so I wouldn't have any problems with the wire catching on a chair or something like that" (P1). 
When asked about how they perceived "being known" by the nurse, one participant explained how the nurse monitored his blood pressure to coordinate when to take his anti-hypertensive medication: "I don't take it anymore [before chemo] ... so now my blood pressure's a little high, so she got nervous, so she called downstairs to Dr. --- who's my doctor and he said don't worry about it, just keep checking" (P7).

Timely provision of information and answers to questions were noted by participants as important aspects of receiving person- and situation-responsive care. One participant shared: "They know that you're scared. First time I came in here I was petrified and she relaxed me and she just said, "relax" and says this is going to be done, this is going to be done. Just follow her instructions and you'll get to the end" (P1). Similarly, another participant described her first day and what her nurse did to provide information and guidance: "[She was] giving me a lot of information on what to expect in terms of chemo and what to do, what not to do, what I'd feel, making sure, explaining to me all of the drugs and the side effects, and the things I needed to be aware of... At the same time she reassured me in the very beginning that if there's anything you need or any concerns you have, this is my card, this is my number, you can reach me, you can call me and you'll get a call back" (P17).

Additionally, participants noted the timeliness of the nurse providing information and answering questions outside of their clinic visits. One spoke of his experience with contacting his nurse with a question about symptom management: "Last week I had a problem and I called up for her and within, let's say within a half an hour she returned my phone call" (P1).

Given that patients interact with a variety of healthcare professionals, many described the importance of seamless communication and its contribution to person- and situation-responsive care. A participant described how his symptoms were communicated to the healthcare team by the nurse: “When I went to see Dr. ---, who's my oncologist, he gave me the file to take to the pharmacy and, uh, in the file I noticed her writing was describing the telephone conversation she had with me, saying I had diarrhea and that I was upset and I was really depressed and she wrote it all down and filled all of the papers so that Dr. --- would see it" (P1).

Furthermore, a participant described how effectively communication about her situation occurred within the nursing team even when her primary nurse was not present: "Even when there was one nurse who was covering for my primary, she already seemed to know me... I said to her, you know, 'usually my IV burns,' and she said, 'Yah, I know, but this drug shouldn't burn you now, because you're not getting the other two'... So, she already had this information, she already knew" (P17).

Another participant described how his concerns about being cared for by different members of the team were supported by his primary nurse: "They must have spent hours [with me] so they knew how to handle me. That helped a lot because I was always very nervous when it wasn't [my nurse]... if they are trained they know. [My nurse would say] 'If I'm not here, you shouldn't be nervous, they will take care of you, one of my colleagues, they will take care of you' " (P19, translated from French).
Participants perceived that they received comprehensive and tailored care and this, in turn, was related to their feelings of "being known." With respect to tailored care, one participant explained how the nurse identified her particular issues with intravenous (IV) therapy and continued to personalize her care during subsequent visits. "She knows the issues of concern, she knows right away when I come that, my IV will be more difficult, so she's already warmed up my arm, she's already done these things in advance, she knows the IV burns, so she already has a secondary line ready... she already knows" (P17).

Similarly, another explained how the nurse began bringing him a cup of water to swallow his pills because of difficulties he had experienced using a water bottle: "I took these pills and I take them with a bottle of water, and I take 20 pills, I take them five at a time, and some of the pills ended up in the bottle of water. Anyway, so, I got, I drank them and everything was fine. And when I came today, she remembered what happened last time, a month ago, and I was very impressed that she actually remembered that" (P7).

\section{Being Acknowledged as a Person with Occupational and Social Interests that Go Beyond the "Sick Role"}

For some $(n=3)$, being acknowledged as an individual beyond their illness impacted their perceptions of "being known". This was demonstrated when providers acknowledged and recalled the patients' social context with respect to family, important milestones and life events, and recognized the patients' social and occupational roles beyond their life with cancer. One participant spoke about the nurse's understanding of her family system: "It's like remembering that, uh, you know, who my girlfriend is, I have a 10-year-old, that kind of stuff is 'being known'” (P5). One participant described the importance of the nurse knowing an important event in his family when his wife was hospitalized. "When my wife came back [the nurse] gave her a big, big hug, and kissed her and held her tight. It's not everybody who will do this" (P1).

With respect to recognition of one's roles outside of cancer care, a participant described how “...it's really important because, you know, you're more than just a patient going through this, you're a person with many different roles... they knew I was a mother of twins, they knew I [was] a wife, a colleague, I have many different roles" (P17).

Similarly, another participant described the importance of having the staff treat him like a normal person: "It's not because I'm sick and that I'm here that I'm a different person you know? I'm like everyone else. Not different... in any case, yes, I'm here, I have long days, five days per week, one week per cycle, but they don't treat you like a sick person-they treat you like a normal person" (P15, translated from French).

\section{DISCUSSION}

In this study participants' narratives of "being known" highlighted the role played by nurses in providing human warmth and caring, tailored competent care, and appreciating patients as individuals beyond their disease. Similar characterizations of "being known" exist in the literature. Jacobsen et al. (2015) 
found that young adults in a cancer care environment felt treated as more than "a number," being recognized as a person with cancer with their unique context of young adulthood. Factors within the cancer care setting such as a welcoming and friendly staff, contributed also to "being known." Additionally, Thorne et al.'s (2005) exploration of patient-provider relationships identified the following salient features of the concept: human connection through physical touch, recognizing the patient as more than their illness and acknowledging the patient's uniqueness. These elements also contributed to patients' overall sense of feeling cared for. In exploring the effects of "being known," Flickinger, Saha, Moore, and Beach (2013) reported that these elements were significantly associated with patient engagement in and adherence to HIV treatment. In addition, these findings demonstrate how key features of "being known" contribute to patients' perceptions of higher quality care.

A major feature of "being known" in the present study had to do with person- and situation-responsive care, as well as mutual disclosure with their primary nurse. Essential to providing responsive care, nurses need to know the patient's unique characteristics, needs, and care preferences and use this knowledge to guide clinical decision making and interventions. In recent literature, situation-responsive care has given way to "knowing the patient", however the premise remains the same (Radwin, 1995; Radwin, 1996; Whittemore, 2000). When healthcare professionals have an in-depth understanding of patients, as individuals, they are better able to tailor their interventions. It is through these interventions-such as coordinating care with the pharmacist, administering treatment to prevent a patient's medication reaction, and providing personalized information-that nurses in the present study demonstrated to their patients that they understood what was important and unique to their care. Indeed, these nursing actions were part of patients' accounts of "being known" and becoming partners in their own care.

Similarly, Radwin (2000) notes that "from the patient's perspective, excellent care was characterized by professional knowledge, continuity, attentiveness, coordination, partnership, individualization, rapport, and caring” (p. 179). More specifically, quality nursing care described by patients include nursing teams' positive attitudes, knowledge and professional skills (Attree, 2001; Rchaidia, Dierckx de Casterlé, De Blaeser, $\&$ Gastmans, 2009). Furthermore, Rchaidia et al. (2009) note that patients perceive high-quality care when nurses are keenly aware of and meeting changes in patient care needs, providing emotional support, demonstrating technical skill proficiency, relating to the patient, as well as creating a caring environment through relatedness. In this study, participants perceived "being known" as an inherent part of quality nursing care.

Likewise, PCC involves the tailoring of care based on individuals' profiles and needs (Arora et al., 2009; Ekman et al, 2011; National Cancer Institute, 2007). McCormack and McCance (2006) specify that PCC involves working with patients' beliefs and values, engaging them in care, sharing decision making, having a sympathetic presence, and meeting physical needs. The Health Innovation Network of South London (2016) details key elements of PCC that are most predictive of positive patient outcomes, including: recognizing individuality; approaching assessment and care holistically; promoting coordination and continuity of care; creating an environment that is physically, culturally, and psychosocially supportive; ensuring a well-trained and communicative healthcare team. Many of these elements are consistent with what participants identified as "being known".

The third concept, being acknowledged as a person with occupational and social interests that go beyond the "sick role", was embedded in some participants' narratives. However, this feature of "being known" has received little attention in previous work. Although the literature on "being known," PCC, and "knowing the patient," addresses the patient's individuality or uniqueness, it is for the purpose of providing responsive care (Flagg, 2015; Radwin, 1995, 1996; Thorne et al., 2005). The present study expanded the meaning of uniqueness and illustrated the connection to patients' perceptions of "being known" by nursing staff. This involved also acknowledging the patient as a person outside of the cancer experience. As such, being recognized for their social and occupational roles, as well as clinical staff acknowledging important life events, can further contribute to perceptions of "being known."

\section{LIMITATIONS}

There are several limitations inherent to this study. The resulting sample was demographically homogeneous, consisting of Caucasian participants who all had partners and a high household income ( $>\$ 100,000)$. This homogeneity could have contributed to similar experiences and needs, which may not accurately reflect individuals with different sociodemographic backgrounds. Furthermore, the rather abstract nature of the concept of "being known" may have made it difficult for patients to grasp and explore meanings associated with the concept.

\section{CONCLUSION}

This study situates "being known" within PCC, as a key factor that contributes positively to patients' healthcare experiences and the crucial role nurses have in delivering PCC. In light of this, nursing management at the study site has implemented nursing rounds to address these needs. Future studies should focus on sociodemographic factors that may affect patients' perceptions of “being known." Further studies should also elucidate how specific nursing interventions such as collaborative nursing rounds or the use of a therapeutic nursing care plan might contribute to feelings of being known.

\section{ACKNOWLEDGEMENTS}

We would like to thank Hope a Cope volunteers for generously dedicating their time for participant recruitment, as well as the JGH Cancer Care treatment centre for their help in coordinating participant interviews. Dr. Ariella Lang, Dr. Margaret Purden, Saima Ahmed and Jacqueline Vachon provided much appreciated feedback on earlier versions of this manuscript. 


\section{REFERENCES}

Arora, N.K., Street, R.L., Jr., Epstein, R.M., \& Butow, P.N. (2009). Facilitating patient-centered cancer communication: A road map. Patient Education a Counseling, 77(3), 319-321 313p. doi:10.1016/j. pec.2009.11.003

Attree, M. (2001). Patients' and relatives' experiences and perspectives of 'good' and 'not so good' quality care. Journal of Advanced Nursing, 33(4), 456-466.

Balogh, E.P., Ganz, P.A., Murphy, S.B., Nass, S.J., Ferrell, B.R., \& Stovall, E. (2011). Patient-centered cancer treatment planning: Improving the quality of oncology care. Summary of an Institute of Medicine workshop. The Oncologist, 16(12), 1800-1805.

Boyatzis, R.E. (1998). Transforming qualitative information: Thematic analysis and code development. Sage.

Brataas, H.V., Thorsnes, S.L., \& Hargie, O. (2010). Themes and goals in cancer outpatient-cancer nurse consultations. European Journal of Cancer Care, 19(2), 184-191.

Ekman, I., Swedberg, K., Taft, C., Lindseth, A., Norberg, A., Brink, E., ... Lidén, E. (2011). Person-centered care-Ready for prime time. European Journal of Cardiovascular Nursing, 10(4), 248-251.

Fillion, L., de Serres, M., Cook, S., Goupil, R.L., Bairati, I., \& Doll, R. (2009). Professional patient navigation in head and neck cancer. Paper presented at the Seminars in Oncology Nursing.

Flagg, A.J. (2015). The role of patient-centered care in nursing. Nursing Clinics of North America, 50(1), 75-86 12p. doi:10.1016/j. cnur.2014.10.006

Flickinger, T.E., Saha, S., Moore, R.D., \& Beach, M.C. (2013). Higher quality communication and relationships are associated with improved patient engagement in HIV care. Journal of Acquired Immune Deficiency Syndromes, 63(3), 362.

Health Innovation Network of South London. (2016). What is personcentred care and why is it important? (pp. 1-5, Publication). South London, UK. Retrieved from http://www.hin-southlondon.org/ resources/what-is-person-centred-care

Jacobsen, S., Bouchard, G., Emed, J., Lepage, K., \& Cook, E. (2015). Experiences of "being known" by the healthcare team of young adult patients with cancer. Oncology Nursing Forum, 42(3), 250-256.

Kruijver, I.P., Kerkstra, A., Bensing, J.M., \& van de Wiel, H.B. (2000). Nurse-patient communication in cancer care. A review of the literature. Cancer Nursing, 23(1), 20-31.
Kvåle, K., \& Bondevik, M. (2008). What is important for patient centred care? A qualitative study about the perceptions of patients with cancer. Scandinavian Journal of Caring Sciences, 22(4), 582-589.

Mazor, K.M., Beard, R.L., Alexander, G.L., Arora, N.K., Firneno, C., Gaglio, B., ... Gallagher, T.H. (2013). Patients' and family members' views on patient-centered communication during cancer care. Psycho-Oncology, 22(11), 2487-2495. doi:10.1002/pon.3317

McCormack, B., \& McCance, T.V. (2006). Development of a framework for person-centred nursing. Journal of Advanced Nursing, 56(5), 472-479.

National Cancer Institute (2007). Patient-centered communication in cancer care: Promoting healing and reducing suffering (07-6225). Bethesda, MD.

Pomney, M-P., Ghadiri, D.P., Karazivan, P., Fernandez, N., \& Clavel, N. (2015). Patients as partners: A qualitative study of patients' engagement in their health care. PLoS ONE, 10(4), e0122499.

Radwin, L.E. (1995). Knowing the patient: a process model for individualized interventions. Nursing Research, 44(6), 364-370.

Radwin, L.E. (1996). 'Knowing the patient': A review of research on an emerging concept. Journal of Advanced Nursing, 23(6), 1142-1146.

Radwin, L.E. (2000). Oncology patients' perceptions of quality nursing care. Res Nurs Health, 23(3), 179-90.

Rchaidia, L., Dierckx de Casterlé, B., De Blaeser, L., \& Gastmans, C. (2009). Cancer patients' perceptions of the good nurse: A literature review. Nursing Ethics, 16(5), 528-542.

Thomsen, D.K., Pedersen, A.F., Johansen, M.B., Jensen, A.B., \& Zachariae, R. (2007). Breast cancer patients' narratives about positive and negative communication experiences. Acta Oncologica, 46(7), 900-908 909p.

Thórarinsdóttir, K., \& Kristiánsson, K. (2013). Patients' perspectives on person-centred participation in health care: A framework analysis. Nursing Ethics, 0969733013490593.

Thorne, S.E., Kuo, M., Armstrong, E.-A., McPherson, G., Harris, S.R., \& Hislop, T.G. (2005). Being known: patients' perspectives of the dynamics of human connection in cancer care. PON PsychoOncology, 14(10), 887-898.

Whittemore, R. (2000, Jan. 1). Consequences of not "knowing the patient". Clinical Nurse Specialist, 14(2), 75-81. 